\title{
Answer to a Quiz: ECG-Pacemaker on page 26 and case discussion
}

Answer: Correct answer - 4

In this patient with moderate left ventricular ejection fraction depression at $45 \%$, and permanent atrial arrhythmia with fast ventricular response, despite several radiofrequency ablation procedures, a strategy of atrioventricular node ablation and His bundle pacing (as an alternative to biventricular pacing) has been proposed. His bundle pacing has just been integrated in the last American guidelines on pacing in 2018 (1). In this specific setting, no atrial lead is then needed. At the beginning of the experience, some centers are adding a back-up ventricular lead, in case of Hissian lead dysfunction or dislodgement (pacemaker-dependent patient). This was the case in this patient. The landmark of selective His bundle capture is the identical QRS morphology between native QRS and paced-QRS complexes (Fig. 1).

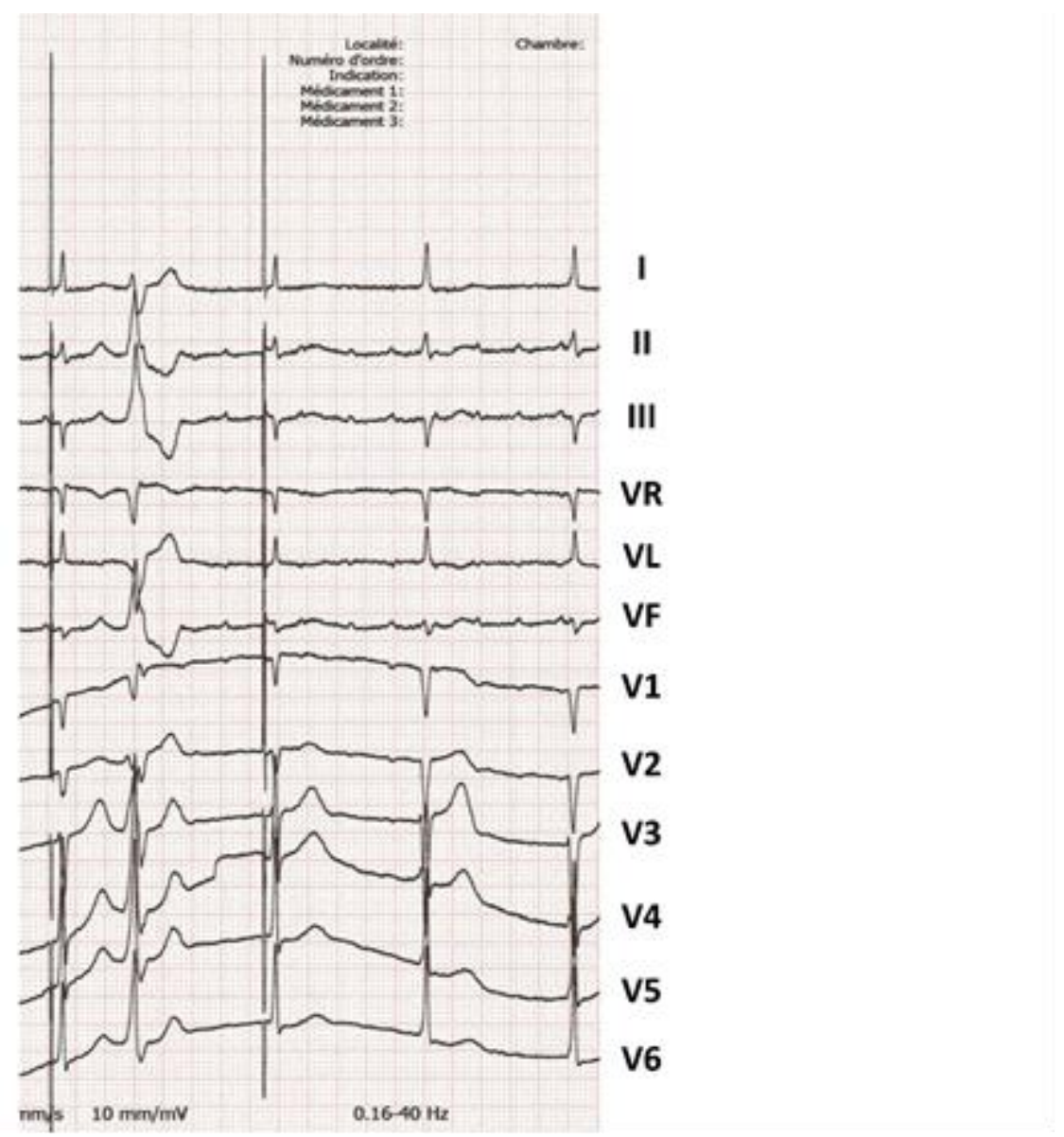

Figure 1. Selective His bundle capture in VVI mode showing identical paced QRS morphology with native QRS from the patient 
1. An incorrect lead connection may be suggested with the Hissian lead connected in the atrial port and the atrial lead connected in the ventricular port. The first two beats of the tracing are seen before the application of the magnet, corresponding to a VVI mode (or a conventional DDD programming including then an atrial and a ventricular lead, with a switching mode into a VVI mode because of the atrial arrhythmia). It would then be impossible to observe a single spike in the presence of a DDD mode (with incorrect lead connection) because the spike is followed by a ventricular complex, and one would see a second spike after the paced atrioventricular delay.

A normal magnet function for a His bundle pacing with right ventricular (RV) back-up lead is that first pacing spike is followed by His capture, the second spike is the RV back-up pacing, but not capturing as $R V$ is still refractory. If leads were reversed: wide QRS complexes (from RV pacing) followed by a second pacing spike without ventricular/His capture (or delayed retrograde atrial capture via atrioventricular node) would be seen.

2. The second spike cannot induce any paced ventricular complex because falling into the ventricular refractory period, the ventricles having just been depolarized by the first paced QRS complex.

3. It is unlikely that all the paced ventricular complexes are fused with native spontaneous QRS complexes. The patient has an atrial arrhythmia with no relationship between the $P$ waves and QRS complexes (atrioventricular node ablation), thus fusion is excluded.
4. The first spike is followed by a narrow paced-QRS complex suggesting a selective His-bundle pacing capture. The second spike is due to the back-up ventricular lead that was implanted, not inducing any captured beat because falling into the ventricular refractory period (Fig. 2).

5. There is no obvious distinguishable or individualized sinus $P$ wave. $P$ waves are at a non-physiologic rate as seen in lead II (approximately 300 beats per minute), consistent with an atypical atrial tachycardia.

\section{Sok-Sithikun BUN ${ }^{1}$, Abdelkarim Errahmouni ${ }^{2}$ \\ ${ }^{1}$ Pasteur University Hospital, Nice, France \\ ${ }^{2}$ Dupuytren University Hospital, Limoges, France}

Peer-review: Internal

Conflict of interest: None to declare

Authorship: S.S.B. and A.E. equally contributed to preparation of quiz

Acknowledgement and funding: None to declare

\section{References}

1. Kusumoto FM, Schoenfeld MH, Barrett C, Edgerton JR, Ellenbogen KA, Gold MR, et al. 2018 ACC/AHA/HRS guideline on the evaluation and management of patients with bradycardia and cardiac conduction delay: A Report of the American College of Cardiology/American Heart Association Task Force on Clinical Practice Guidelines and the Heart Rhythm Society [published correction appears in Circulation. 2019 Aug 20;140(8):e506-e508]. Circulation 2019; 140: e382-e482. 


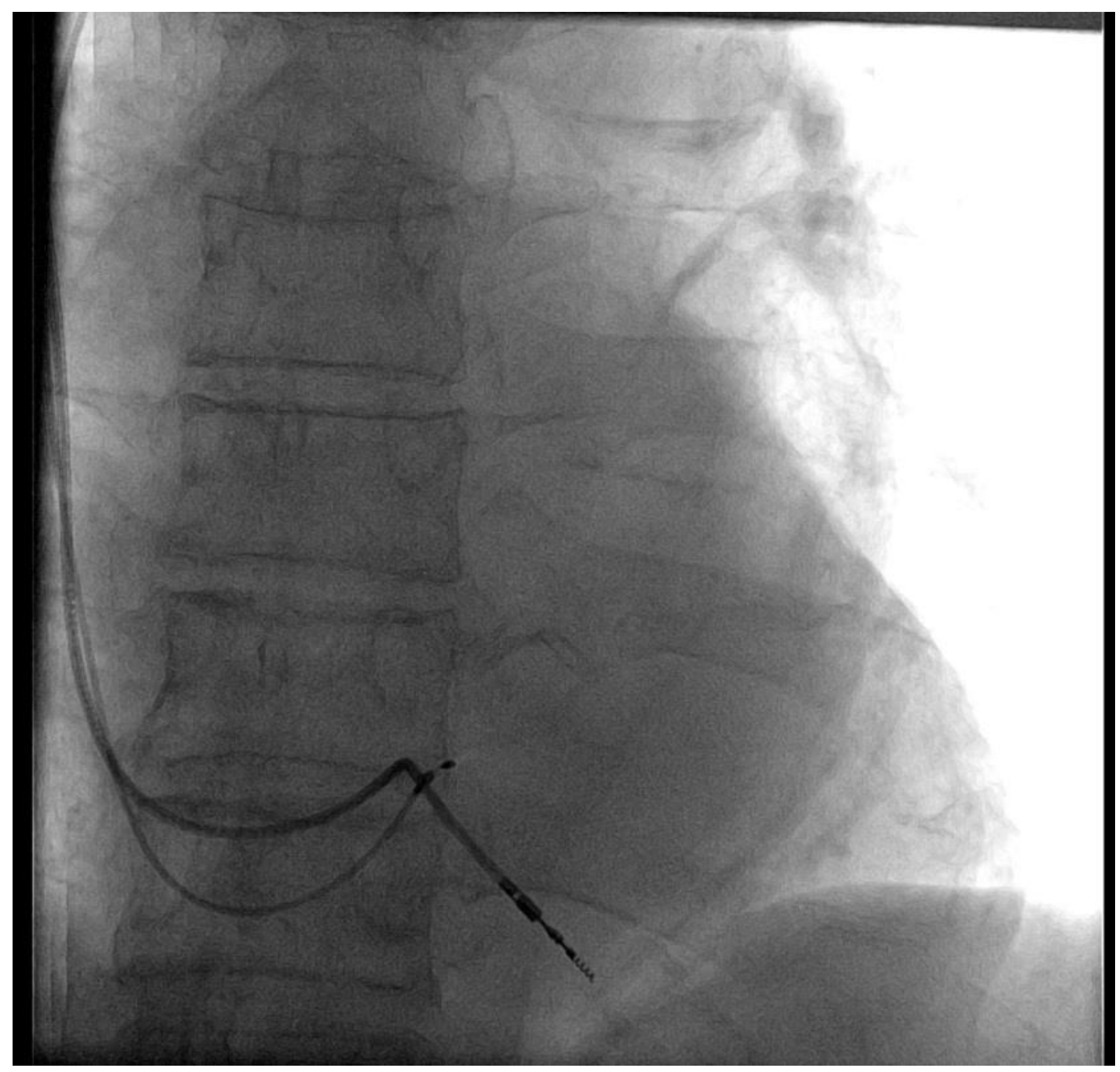

Figure 2. Fluoroscopic antero-posterior projection showing the Hissian lead with ventricular back-up lead 